Audiology

Neurotology
Audiol Neurotol 2020;25:79-90

DOI: $10.1159 / 000503600$
Received: August 7, 2019

Accepted: September 16, 2019

Published online: December 4, 2019

\title{
Chronic Electrical Stimulation of the Otolith Organ: Preliminary Results in Humans with Bilateral Vestibulopathy and Sensorineural Hearing Loss
}

\author{
Angel Ramos Macias ${ }^{b}$ Angel Ramos de Miguel ${ }^{c}$ Isaura Rodriguez Montesdeoca ${ }^{a}$ \\ Silvia Borkoski Barreiro ${ }^{a}$ Juan Carlos Falcón González ${ }^{a}$

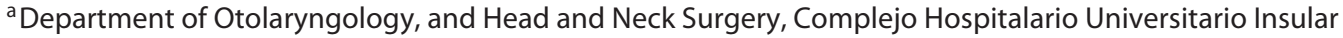 \\ Materno Infantil de Gran Canaria, Las Palmas, Spain; ${ }^{b}$ Department of Otolaryngology, Faculty of Medicine, \\ University of Las Palmas de Gran Canaria, Las Palmas, Spain; ${ }^{C}$ Hearing and Balance Laboratory, University of \\ Las Palmas de Gran Canaria, Las Palmas, Spain
}

\author{
Keywords \\ Vestibular implant $\cdot$ Balance $\cdot$ Utricle $\cdot$ Saccule $\cdot$ Bilateral \\ vestibulopathy
}

\begin{abstract}
Introduction: Bilateral vestibulopathy is an important cause of imbalance that is misdiagnosed. The clinical management of patients with bilateral vestibular loss remains difficult as there is no clear evidence for an effective treatment. In this paper, we try to analyze the effect of chronic electrical stimulation and adaptation to electrical stimulation of the vestibular system in humans when stimulating the otolith organ with a constant pulse train to mitigate imbalance due to bilateral vestibular dysfunction (BVD). Methods: We included 2 patients in our study with BVD according to Criteria Consensus of the Classification Committee of the Bárány Society. Both cases were implanted by using a full-band straight electrode to stimulate the otoliths organs and simultaneously for the cochlear stimulation we use a perimodiolar electrode. Results: In both cases Vestibular and clinical test (video head impulse test, videonistagmography cervical vestibular evoked myogenic potentials, CVEMP and oVEMP), subjective visual vertical test, computerized dynamic posturography,
\end{abstract}

dynamic gait index, Time UP and Go test and dizziness handicap index) were performed. Posture and gait metrics reveal important improvement if compare with preoperartive situation. Oscillopsia, unsteadiness, independence and quality of life improved to almost normal situation. Discussion/Conclusion: Prosthetic implantation of the otolith organ in humans is technically feasible. Electrical stimulation might have potential effects on balance and this is stable after 1 year follow-up. This research provides new possibilities for the development of vestibular implants to improve gravitoinertial acceleration sensation, in this case by the otoliths stimulation.

\footnotetext{
(C) 2019 The Author(s)

Published by S. Karger AG, Basel
}

\section{Introduction}

The main functions of vestibular system are to adjust head, neck, and trunk positions to maintain balance and gait and to adjust head and neck movements to maintain images at the fovea.

Bilateral vestibulopathy (BVP) or bilateral vestibular hypofunction is an important cause of imbalance that is frequently misdiagnosed. Individuals with BVP report

\begin{tabular}{ll}
\hline KARGER & $\begin{array}{l}\text { (c) } 2019 \text { The Author(s) } \\
\text { Published by S. Karger AG, Basel }\end{array}$ \\
E-Mail karger@karger.com & $\begin{array}{l}\text { This article is licensed under the Creative Commons Attribution- } \\
\text { NonCommercial-NoDerivatives 4.0 International License (CC BY- } \\
\text { NC-ND) (http://www.karger.com/Services/OpenAccessLicense). } \\
\text { Usage and distribution for commercial purposes as well as any dis- } \\
\text { tribution of modified material requires written permission. }\end{array}$
\end{tabular}

Prof. Angel Ramos Macias

Department of Otolaryngology, Faculty of Medicine

University of Las Palmas de Gran Canaria, Avda Maritima del Sur SN

ES-35016 Las Palmas (Spain)

E-Mail ramosorl@idecnet.com 
experiencing chronic unsteadiness and vertigo when being in an upright position and walking, and these symptoms may become more severe with head motion and darkness. Bilateral vestibular dysfunction (BVD) has a prevalence of 16-28 cases per 100,000 individuals and is important in the elderly, as it produces more secondary effects in this group. Despite their severe impact on patients' daily life, vestibular function cannot be restored in a high number of patients, and vestibular implants (VI) improve the health state of only some patients. Many studies carried out in animals and humans lead to the concept that restoring vestibular function by electrically stimulation of the vestibule can be useful. At this point, most of the research has been focused on developing VI to be placed in the semi-circular canal ampullae. However, no attempt has been made to chronically stimulate the otolith organs in humans [Sprinzl and Riechelmann, 2010; Ward et al., 2013; Della Santina et al., 2017; Hedjoudje et al., 2019].

The clinical management of patients with bilateral vestibular loss remains difficult, as there is no clear evidence of an effective treatment. Additionally, it must be considered that many dizzy patients may suffer from different conditions that must be considered during their evaluation and treatment.

The otolith organ consists of 2 areas: the utricular sac and the saccular sac. Each of these sacs contains a plate of specialized receptor hair cells and connective tissue called macula. The utricular and saccular maculae are placed approximately perpendicular to each other. The saccule and utricle respond to linear acceleration and to gravity: the saccule senses acceleration in the sagittal plane and the utricle senses acceleration in the horizontal plane. With the head erect (head-up position), the utricular macula is tilted by about 30 degrees (open anterior) with respect to the horizontal plane of the head, whereas the saccular macula is nearly vertical. The spontaneous activity of the primary otolithic afferents is highly regular in some afferents, whereas it is highly irregular in others. In addition, there is a continuum of regularity between these extremes. Physiological responses are closely related to this dimension of regularity. Irregular neurons show a strong response to changes in linear accelerations, while regular neurons show a faithful response to maintained linear accelerations [Goldberg and Fernandez, 1984; Curthoys et al., 2018].

The superior vestibular nerve carries impulses from the utricle and semicircular canals to the vestibular nucleus, and then, these impulses travel through the medial longitudinal fasciculus mainly to the oculomotor nucle- us. It stimulates mainly the oculomotor nucleus and the medial rectus and inferior oblique. The inferior vestibular nerve carries impulses from the saccule to the medial vestibular nucleus and is related to the vestibulospinal tract, which carries these impulses to the spinal accessory nucleus. This nucleus innervates the sternocleidomastoid and the vestibulo-collic reflex related to maintain neck musculature in relation to head position [Brodal, 1974].

For the purpose of this study, and focusing on surgical aspects, it is also important to understand the anatomical relation of the otolith organ and the stapedial footplate. With respect to the distances from the stapedial footplate to the vestibular end organs and cochlear duct, they range from 1.9 to $2.4 \mathrm{~mm}$ to the utricle, and from 1.7 to $2.1 \mathrm{~mm}$ to the saccule, while the distance between the cochlear duct and the inferior border of the footplate is around 0.2 mm [Pauw et al., 1991].

The vestibular electrical stimulation tries to produce "artificial" neural patterns to the central nervous system that are similar to those coded by the normal system. In healthy subjects, the vestibular nerves fire spontaneously at a rate of about 90 action potentials per second in the absence of movement $(15,16)$. Electrical stimulation of the utricular nerve in cats causes a distinct pattern of eye movement: a torsion of both eyes in which the upper poles of the eyes roll away from the side being stimulated because of the activation of the contralateral inferior oblique and ipsilateral superior oblique muscles. Complementary, studies have shown that unilateral section of the vestibular nerve causes torsion of both eyes toward the operated side. In natural head movements, the otoliths are activated and generate compensatory eye and postural responses [Curthoys et al., 1991].

Another interesting aspect of this VI research is to generate evidences on vestibular function research, particularly by providing selective and direct access to the otolith end organs structures.

\section{Objective}

The objective is to analyze the effect of chronic electrical stimulation and the adaptation to electrical stimulation of the vestibular system in humans when the otolith organ is stimulated with a constant pulse train to mitigate imbalance due to BVD.

Preliminary results will be presented regarding vestibular testing and clinical aspects, and mainly focused on spatial orientation, balance, posture and gait of 2 patients with otolith chronic electrical stimulation. 
Table 1. Clinical data of both vestibular implanted patients

\begin{tabular}{llllllll}
\hline Patient & Gender & Aetiology & Onset age, years & Age at VI & Side & N elect & Contralat. CI \\
\hline VI/CI-1 & Male & Meningitis & $45(2017)$ & 46 & OI & 3 & $\begin{array}{l}\text { Nucleus }^{\circledR} \text { Profile }^{\text {TM }} \text { Contour }^{\text {Advance }}{ }^{\circledR}-\text { CI512, (2017) } \\
\text { VI/CI-2 }\end{array}$ \\
\hline
\end{tabular}

\section{Material and Methods}

The current study was conducted with the approval of the Ethics Committee of our hospital (CEIm-CHUIMI 2017/956) and performed in accordance with the 1964 Helsinki Declaration and its later amendments or comparable ethical standards. All patients provided written informed consent before participating. All the procedures involving human participants were in accordance with the ethical standards of our institutional research committee.

Two patients matching the following criteria were included in our study: being older than 18 years, presenting BVD according to Criteria Consensus of the Classification Committee of the Bárány Society. In both cases, supervised rehabilitation sessions, in addition to a daily home exercise program, were used before their implantation for more than 1 year. Both cases failed to the adequate vestibular rehabilitation program and also in both cases, a profound hearing loss was found.

The exclusion criteria for this study were the following: being unable to provide consent personally, not matching cochlear implantation criteria, ossification or other inner ear anomalies that prevent full insertion of electrodes, retrocochlear or central origins of hearing impairment, medical contraindications for surgery, chronic depression, dementia and cognitive disorders, cerebellar ataxias without BVP, downbeat nystagmus syndrome, peripheral neuropathies, Parkinson's disease, atypical Parkinson's syndromes, multiple system atrophies, central gait disorders due to normal pressure hydrocephalus, frontal gait disorders, lower-body Parkinson, subcortical vascular encephalopathy or multiple sclerosis.

Subject 1 (vi/ci-1) is a man who had meningococcal meningitis for 1 year before surgery and the implantation was performed at the age of 46 in the left ear. Subject 2 (vi/ci-2) was a man who had meningitis for 15 year before surgery and received the implant in the left ear at the age of 45 .

The first patient received a cochlear implant (CI) in the contralateral ear to be treated 14 months before $\left(\mathrm{CI} 532^{\circledR}\right)$, and the second, 14 years before (Cochlear CI24M ${ }^{\circledR}$ ). In both cases, the left side was implanted with a Cochlear CI $532{ }^{\circledR}$ for cochlear stimulation (CI; although the results are out of the focus of this study) and a Straight Cochlear 24RE ST ${ }^{\circledR}$ for Otolith organ stimulation (VI), in the same surgical procedure simultaneously. The first implantation was performed in July 2018 and the second one in November 2018 (Table 1).

\section{Inclusion Criteria}

Both cases had BVP. For the diagnosis of BVP, we followed the Diagnostic Criteria Consensus document of the Classification Committee of the Bárány Society (21). For the diagnosis of BVP, the horizontal angular vestibulo-ocular reflex (VOR) gain on both sides should be $<0.6$ (angular velocity $150-300^{\circ} /$ s). For the diagnosis of probable BVP, the above-mentioned symptoms and a bilaterally pathological bedside HIT are required.

\section{Radiological Evaluation}

Computed tomography (CT): images were acquired by cone beam CT (RayScan M., Ray Co Ltd., Korea). Cone beam CT has been previously validated as a valuable tool for the assessment of electrodes post-cochlear implantation, as it requires less irradiation than a regular CT and shows less sensitivity to metallic artifacts. Thus, it represents a better method to identify electrode placement in the cochlea and otolith organ. We use this technique in both patients to measure the vestibule size. OsiriX software was used to collect all the images, in the DICOM file format, of the vestibule. A MatLab script was developed to extract and reconstruct the volume of the vestibule. The vestibule depths were $2.79 \mathrm{~mm}$ for 1 patient and $2.53 \mathrm{~mm}$ for the other patient.

An MRI-scan, T1 and T2 images of the cerebellopontine angle, with intravenous gadolinium contrast was performed in order to evaluate the patency of the labyrinth and nerve structure analysis. Inner ear fluid space images and positive perilymph/positive endolymph images were acquired by using a 1.5-tesla unit. Removal of the magnet of the contralateral side was not required.

Three-dimensional (3-D) images were constructed semiautomatically by using both anatomical and tissue information and by fusing the 3-D images of the inner ear fluid space. Custom made software was developed to construct semi-automatically 3-D meshes of the inner ear of the patient and the vestibular electrode array. The reconstruction procedure was based on the following 5 stages:

1. Inner ear location.

2. Volumetric reconstruction of bonny structures.

3. Volumetric reconstruction of electrode.

4. Cleaning mesh manually.

5. Superimpose anatomical structures and electrode array.

For that proposal, OsiriX MD [Rosset et al., 2004] software was used for the volumetric reconstructions of the different structures. MeshLAB [MeshLab 2008] software was used to clean the meshes.

\section{Surgical Procedure}

Stimulation Technique and Surgical Approach

A full-band straight electrode, CI24RE (ST), from Cochlear Ltd (Lane Cove, NSW, Australia) was used. This electrode array has a diameter of $0.4 \mathrm{~mm}$ on the apical part. Each electrode has a cylindrical band of $0.3 \mathrm{~mm}$ width and $0.4 \mathrm{~mm}$ diameter on the tip. The interelectrode space is $0.2 \mathrm{~mm}$ on each lead. Full-band electrodes were selected to assure that the electrodes were facing the closest area of neural tissue related to the saccular area. For the cochlear stimulation, a Cochlear CI532 ${ }^{\circledR}$ (Cochlear Ltd., Sydney, NSW, Australia) 
Fig. 1. Surgical approach and position of both receptors: vestibular and cochlear implant. Incision and anatomical situation of both receptors. a Vestibular implant. b Cochlear implant.
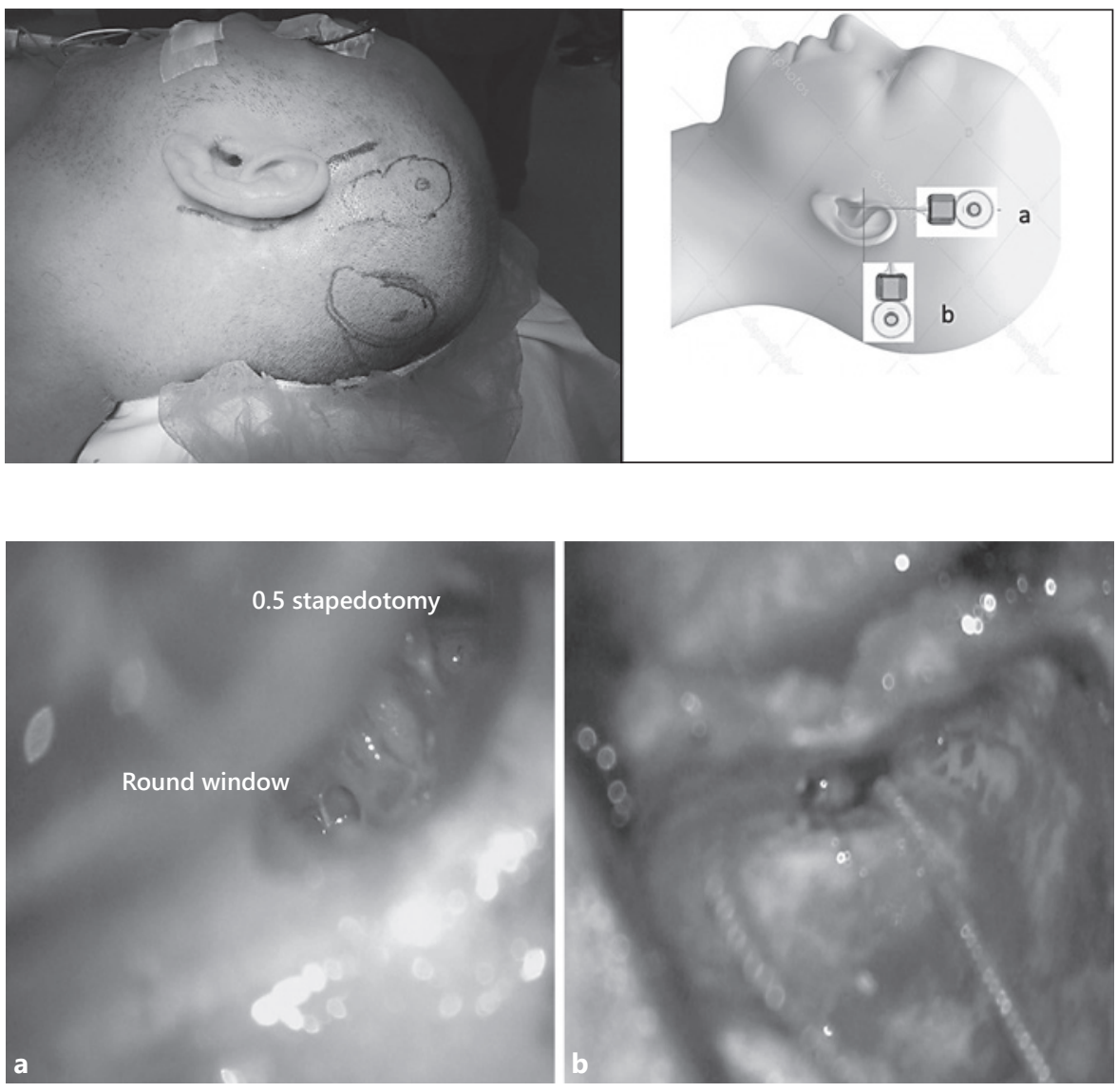

Fig. 2. Anatomical landmarks for vestibular and cochlear implant insertion. Posterior tympanotomy view. a Stapedotomy for VI insertion and Round Window approach for CI. b Vestibular and cochlear implants arrays insertion.
During the surgical procedure, the facial nerve was monitored with the Nerve Integrity Monitor 2tm system (Medtronic, Minneapolis, MN, USA).

Fixation of both implants was made independently, and a "Three-point fixation" of the VI electrode array (Stapedotomy, fossa incudes, and cortical edge of mastoidectomy) was performed. No immediate postsurgical vertigo was observed. The postsurgical hospital stay was not longer than that after CI surgery. During the postsurgical stay, CT scans and 3-D reconstruction were performed to check the placement and orientation of both implants, aiming at the proximity to the lower vestibular nerve (Fig. 3).

\section{Intraoperative Testing}

As previously described [Ramos de Miguel et al., 2017] a "vestibular response telemetry" (VRT) software was designed by using the nucleus implant communicator library (Cochlear LTD) for Python (Python Software Foundation, version 2.4) in order to obtain electrically evoked action potentials (ECAPs) from the vestibular nerve. The software automatically evaluates all of the configurations available to find the best ECAP response. On average, total acquisition takes $10 \mathrm{~min}$. The software communicates then with the speech processor to capture, process, and store the measurement data on a computer. The VRT software controls the parameters of the stimulus used to evoke and record the response to be measured intraoperatively. 

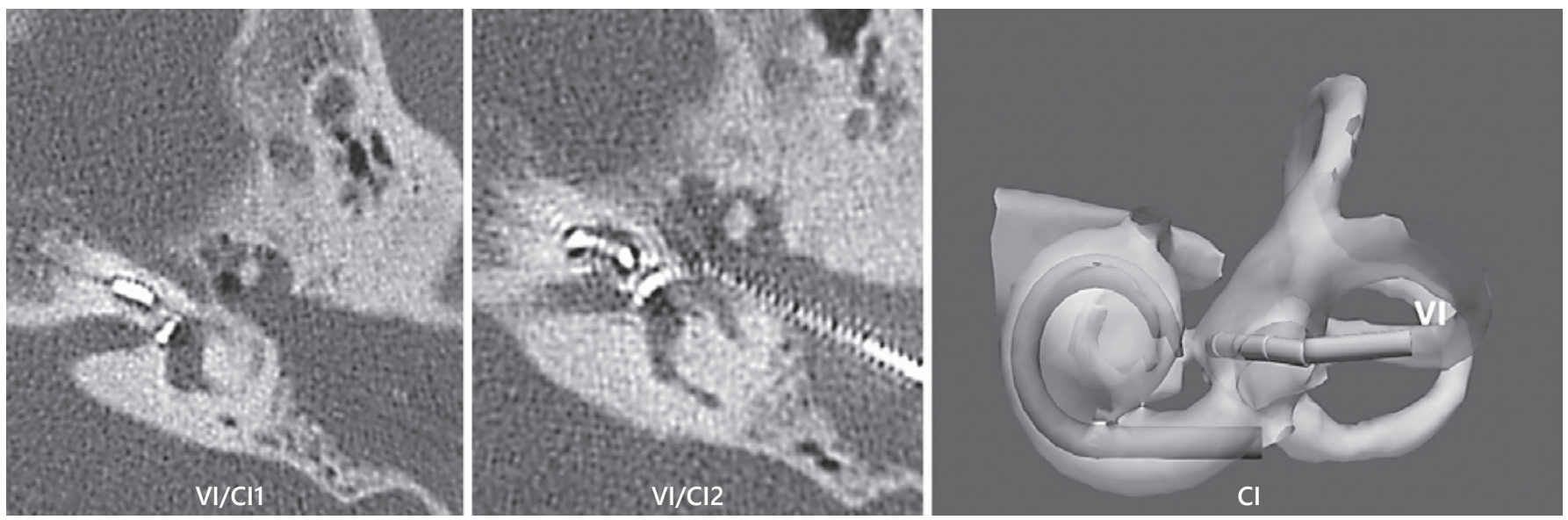

Fig. 3. Final position of the cochlear and vestibular implant in the CT images and 3D reconstruction. CT scan showing the insertion of vestibular and cochlear electrode arrays in both patients (VI/CI1 and VI/CI2). Also, it shows a 3 D reconstruction of the final situation in VI/CI2 subject. VI, vestibular implant; CI, cochlear implant.

Fig. 4. Vestibular-evoked potential. Neural response in the electrodes. The intraoperative ECAP response obtained in both patients.

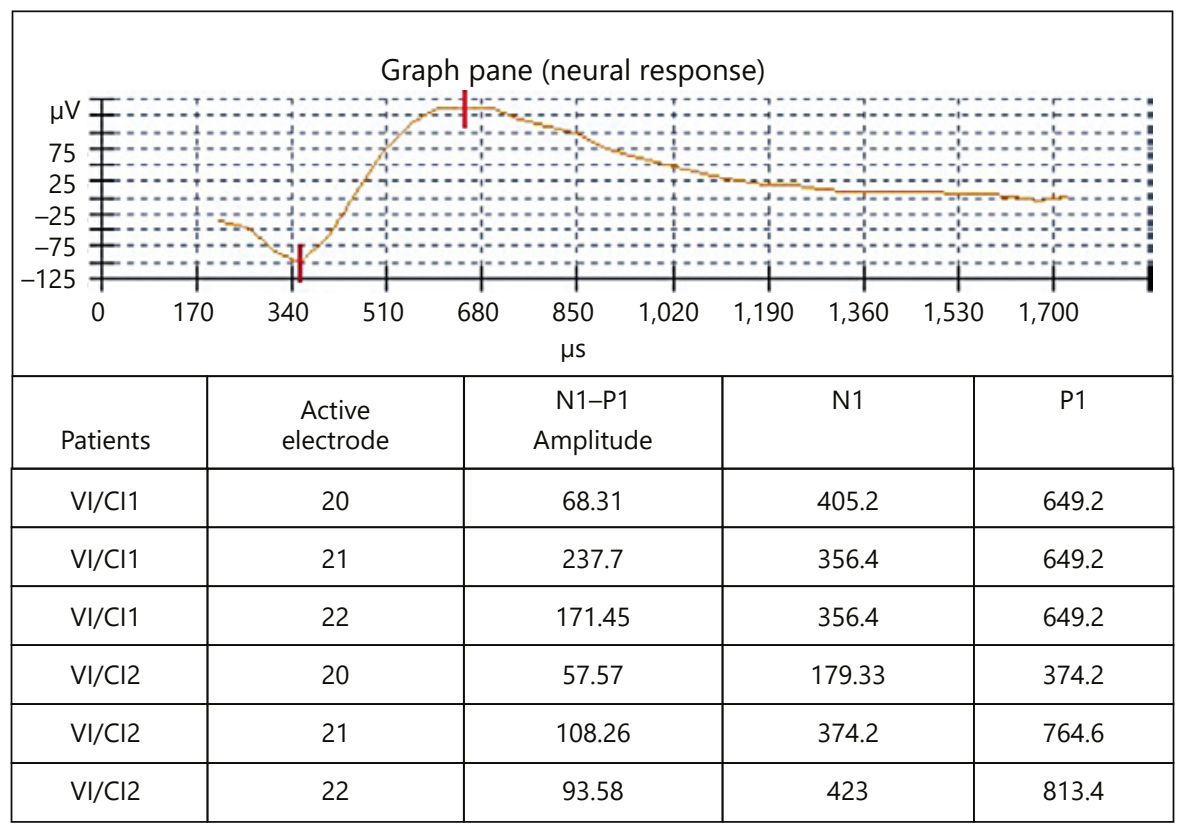

The VRT measurements were done after insertion of the 3 electrodes into the vestibule, and the 2 reference electrodes were correctly placed and covered by tissue. An extracochlear ball electrode on a flying lead was placed under the temporalis muscle (MP1), and a plate electrode was placed on the receiver-stimulator (MP2). Monopolar stimulation (MP) was used in all tests. We then studied the effect of different test parameters, including stimulation rate, number of repetitions, measurement delay, masker level, and masker advance. The specific test (stimulation and recording) parameters studied were the following: (1) measurement delay ( $80-120$ is); (2) stimulation rate $(90$ or $70 \mathrm{~Hz})$; (3) number of samples; and (4) number of averages $(50,100$, or 150$)$. The setup consists of a lap computer, cochlear POD interface, Nucleus
Freedom processor (Cochlear Corp., Sydney, NSW, Australia), and CI24RE (ST).

Additionally, electrically ocular vestibular evoked myogenic potentials (eoVEMP) responses were recorded by using a recording system (Eclipse Interacoustic, EP25). This test was included in order to confirm the stimulation of the vestibular pathway by recording electrically eoVEMPs, by using a trigger system.

To minimize the stimulation artifacts, the developed software implements the forward-masking paradigm and involves a sequence of conditions: probe-only (A), masker followed- by-probe (B), masker-only stimuli (C), and no stimulation (D). The probeonly condition yields the desired neural response plus an artifact from the probe. The masker-and-probe condition with an appro- 
priate masker advance yields stimulus artifacts for both masker and probe, and the neural responses to the probe are absent or decreased because of the forward masking. The masker-only condition yields only the masker artifact. After the recordings of each of these 4 stimulation conditions were performed, extraction of the ECAP from the stimulus artifact was accomplished by using the subtraction method $(A,[B,(C, D)])$ to cancel the large masker stimulus artifacts found in each condition, and this allows one to extract the relatively small neural response.

In order to obtain the neural responses, a second test using Cochlear's Custom Sound Evoked Potential Software tool (version 5.2) was used. The basic parameters used to create the stimulus in channels 20-21 and 22 of the electrode beam were the following: Recording active electrode Prb Act E + 2; Probe Indifferent Electrode MP1; Probe current level (CL) $\geq 200$; Probe Pulse Width 40 $\mu$ s; Probe Rate $40 \mathrm{~Hz}$; Recording Indifferent Electrode MP2; Gain $50 \mathrm{~dB}$; Delay $122 \mu \mathrm{s}$; Number of Sweeps 50; and Measurement Windows 1,600 $\mu$ s (Fig. 4).

\section{Electrically Ocular Vestibular Evoked Myogenic Potentials}

In order to record eoVEMPs (responses attributed to the utricular-ocular reflex pathway) simultaneously with the vestibular ECAP, electrical oVEMPs were performed intraoperatively. A trigger synchronized both systems. In this study, ocular vestibular-evoked myogenic potentials were obtained from both participants by using Eclipse EP 15/EP25/VEMPS (Interacoustics AS, Assens, Denmark system). Responses were collected and analyzed by using a 2-channel surface electrode montage and Eclipse Interacoustic EP25 4.4 VEMP 4.3 recording platform. In order to determine the accuracy of the calibration method, the active electromyogram (EMG) electrode was placed over the medial inferior oblique muscle edge contralateral to the stimulated ear, and the reference electrode was placed on the cheek $1 \mathrm{~cm}$ below. Then montage ground electrode was placed on the mid-forehead. Impedance was kept below $5 \mathrm{kOhms}$.

EMG signals were band pass filtered $(1-3,000 \mathrm{~Hz})$ and recorded in a 25-50 ms window relative to stimulus onset. No online artifact rejection was used. For all eoVEMPs tests, 2 or more trials (100 sweeps each) were obtained. When such responses were not identified after 2 trials, testing was ended. The maximum intensity levels were set from 120 to $220 \mathrm{CL}$, with a $20 \mathrm{CL}$ stepsize, for both single 57 ì biphasic electric pulses (25 ìs/phase with a 10 ì interphase gap). The eoVEMPs tests were first conducted by using electrode 22 and then were repeated by using the other inserted electrodes 21 and 20.

The facial nerve was monitored in all the subjects, and no cross stimulation of the facial nerve was observed in any of these cases.

\section{Postsurgical Measurement and Processor Activation}

During the activation process, both patients described a feeling of surprise, then a sensation of comfort and stability while they stay seated when the activation process was performed. When the increase of the stimulation sensation was excessive, they felt unexpected motion sickness without nausea.

Vestibular stimulation is composed of a constant pulse train of the 3 inserted electrodes. This makes the use of external sensor unnecessary even when the patient is in dim light. The objective of this stimulation is to provide a vestibular activity that masks the absence of stimulus consistent with the forces perceived by the patient and allows the rest of the balance system (vision and proprioception) to maintain balance.
Table 2. Data of first programming session in both patients

\begin{tabular}{llllll}
\hline Patient & $\begin{array}{l}\text { Active elec- } \\
\text { trode }\end{array}$ & NRT & C level & T level & Maxima \\
\hline VI/CI1 & 20 & 182 & 130 & 129 & 8 \\
VI/CI1 & 21 & 182 & 130 & 129 & 8 \\
VI/CI1 & 22 & 179 & 130 & 129 & 8 \\
VI/CI2 & 20 & 223 & 195 & 194 & 8 \\
VI/CI2 & 21 & 241 & 195 & 194 & 8 \\
VI/CI2 & 22 & 244 & 195 & 194 & 8 \\
\hline
\end{tabular}

We use a monopolar stimulation of the electrodes ICE22, ICE21, and ICE20 against ECE1 + 2. The stimulus is a biphasic pulse of 25 us per phase and 8 us of Inter-Phase gap at a stimulation frequency of $900 \mathrm{pps}$. The dynamic range for each patient is 1 CL (as it will be described afterwards) to achieve a constant stimulation in the 3 electrodes involved. AutoNRT measurement was carried out with the basic parameters of the Cochlear Custom Sound Evoked Potential Software version 5.2.

As standard, an ACE (RE) coding strategy, an MP1 + MP2 stimulation and a maximum of 8 were used, so 8 channels activated (from 15 to 22 ) were kept, maintaining channels from 15 to 19 , CL values threshold $(T)=1$, dynamic range $(\mathrm{RD})=1$, and comfort level $(C)=3$. The channels in which responses were obtained were those used to provoke the stimulus. In both patients, channels 20-21 and 22 were selected, assigning as threshold T the minimum value of subjective perception of the patient $\mathrm{RD}=1$ and $\mathrm{C}=+1$, speed of stimulus of $900 \mathrm{~Hz}$ although we considered changing later to $1,200 \mathrm{~Hz}$ given that in both patients the neural responses were above $200 \mathrm{CL}$, which allowed us to low C levels. In both patients, with respect to channel $22,205 \mathrm{~Hz}$ was assigned to the frequency allocation table.

The same "C" and a RD of 1 value were used in all 3 channels $20-22$. The stimulation was started with a value of $70 \%$ of the value of the postsurgical neural response (this percentage oscillated between 70 and 90\%) monitoring the patient's nystagmus through the Videonystagmography testing. The 3 channels were increased simultaneously in 2 CL step keeping always an RD of 1, until observing with the monitoring the decrease or abolition of the nystagmus. The first MAP was recorded at this level of stimulation, creating 3 MAPS with an increment of +2 CL consecutively in the 3 channels. These MAPs were the ones that the patient had been changing every week during the study period. The 3 channels in both patients were evaluated individually, alternating them by active pairs $(22-20,22-21,21-20)$ and all 3 at the same time. And it was observed that the best selection of channels (where the best results have been obtained) was when 3 channels have been used simultaneously.

The values obtained were used as a reference of indicator of perception of the minimum stimulus perceived by the patient to initiate levels $\mathrm{C}$ in the Programming MAP (Table 2).

CP950 Processor (Kanso Cochlear ${ }^{\circledR}$ ) was used by the patients for daily use (16 h/day) and CP910 processor (Cochlear ${ }^{\circledR}$ ) was used during programming and measurements sessions in the hospital.

For the CI, we used the SD method. In both cases, a CP950 Processor (Kanso Cochlear ${ }^{\circledR}$ ) was used. 


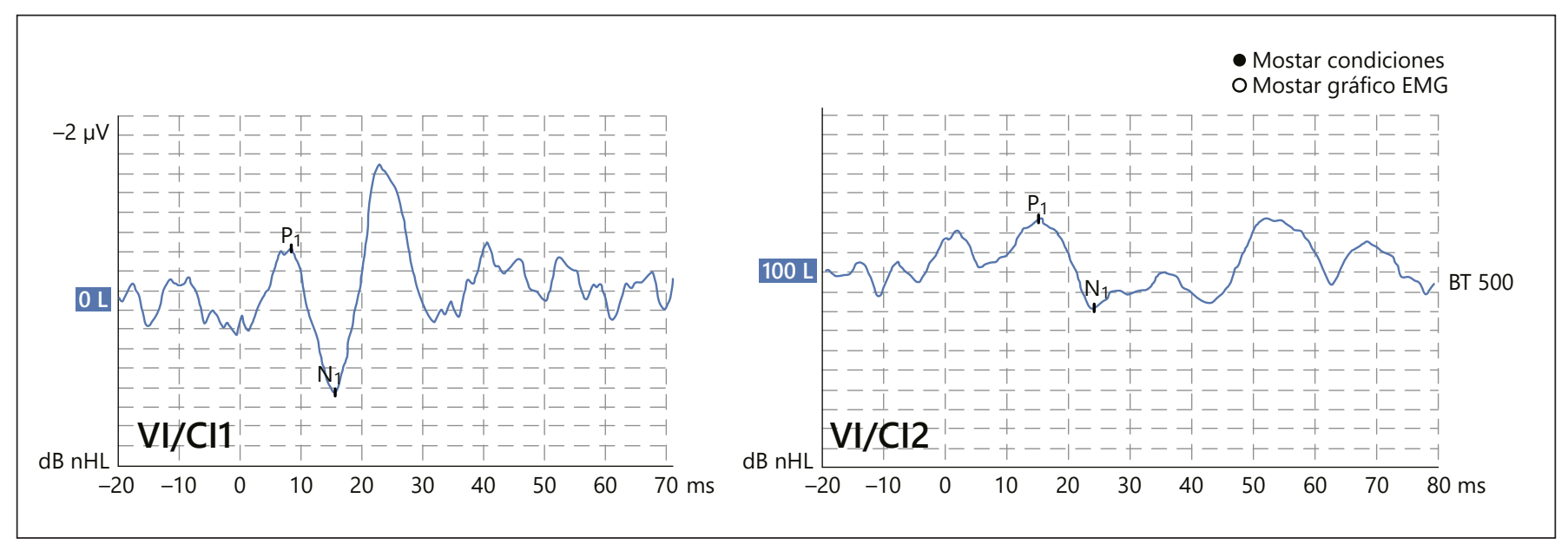

Fig. 5. Vestibular-evoked myogenic potentials. ecVEMPs were obtained from both patients. Results after 1 month postsurgery are presented. VI, vestibular implant; CI, cochlear implant.

\section{Results}

Posture and gait metrics of the patients reveal a mean improvement with respect to their pre-surgery situation. Oscillopsia, unsteadiness, independence, and the resulting quality of life remarkably improved, reaching values close to normality.

\section{Clinical Evaluation}

One month after the surgical procedure was performed, the vestibular and CI were alternately activated. Both subjects showed an immediate response (in a few seconds) after the activation of their VI. After continued use, they reported a perception of change $5 \mathrm{~min}$ after VI turned off and a residual effect for at least $5 \mathrm{~h}$. The tests were re-evaluated to check the results reproducibility with a frequency of a month between sessions. With respect to side effects, only Subject 1 reported a slight increase in his previous tinnitus during VI turned on, but that disappeared after 1 month.

\section{Vestibular Test}

Video Head Impulse Test

Horizontal angular VOR gain on both sides semicircular canals. Bilaterally reduced or absent angular VOR function by bilaterally pathological horizontal angular VOR gain $<0.66$, measured by the video-HIT (ICS Impulse type 1085 from GN Otometrics A/S). Although, predictably, there were no expectations of changes in the VOR gain, as the stimulus was focused on the otolith organ, better VOR gain in the semicircular canals of the implanted side was observed in one of the patients. However, this was not enough to reach a normal value in normal situation, but this data must be analyzed as probably it, together with the excellent clinical response, must be considered in future research.

\section{Videonistagmography}

(VisualEyes $^{\mathrm{TM}} 515 / 525$ by Interacoustics) was used to test spontaneous nystagmus. This test was conducted with the vision-denied cover attached to the front of the mask, eliminating any possibility of fixation. In the second patient, a decrease in the average slow phase velocity using VI (from 2.3 to 0.3 ) was obtained. As an important weakness we missed the initial data of the first patient after activation.

\section{Vestibular Evoked Myogenic Potentials}

Measurements of the vestibular evoked myogenic potentials (VEMPs) allow for an evaluation of the function of otolith organs and are therefore a useful addition to the neurotologic test battery. Two subtypes have been increasingly used in clinical practice as well as in research over the last years: cervical VEMP (cVEMP) and ocular VEMP (oVEMP). Both generated in response to air or bone conduction stimulus. The cVEMP is recorded by EMG electrodes over the tensed sternocleidomastoid muscles, and consists of a short-latency (13 ms from stimulus onset to response peak) positive EMG potential called the cVEMP p13-n23. The oVEMP is a small (5-10 iV) negative potential recorded by electrodes on the skin beneath the eyes when the person looks up. VEMPs can be used to assess otolith integrity 


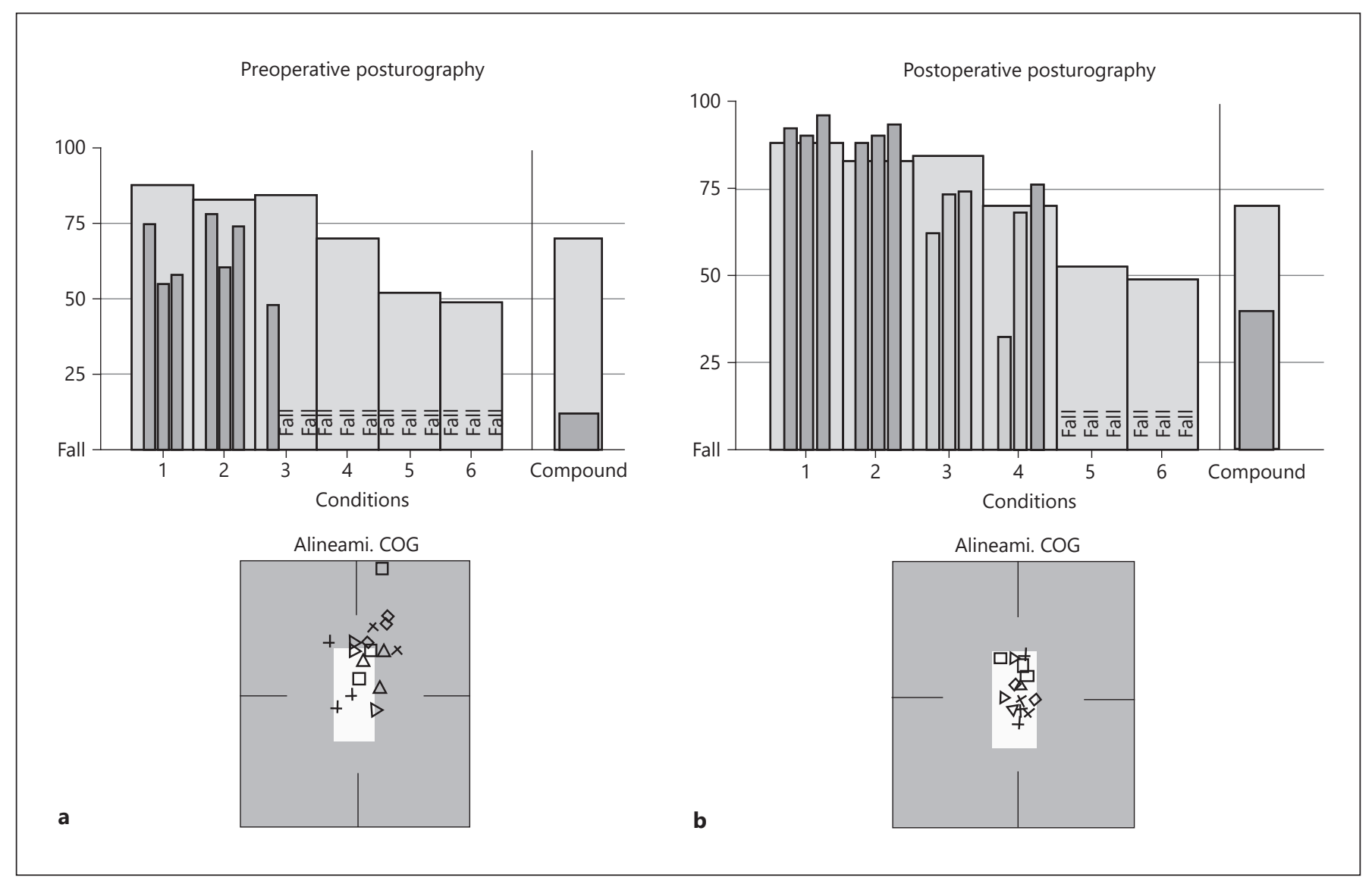

Fig. 6. Response preoperative (a) and postoperative (b) posturography. CDP: LOS with better directional control and increase in maximum excursion. SOT with an improvement in Conditions 1-4 and increase in composite score and center of gravity more centered adaptation test with evident improvement of the reaction control.

as well as vestibulo-collic and vestibulo-occular pathways [Curthoys, 2010].

Cervical and oVEMPs are reflexes mediated by the saccule and utricle respectively. There are data confirming that oVEMPs to $500 \mathrm{~Hz}$ probe utricular function and cVEMPs to $500 \mathrm{~Hz}$ probe saccular function come from the pattern of responses of patients with vestibular neuritis, in which hearing is not affected, but vestibular function is affected [Halmagyi et al., 2010].

In this study, cervical and ocular vestibular-evoked myogenic potentials were obtained from all participants using bilateral air conduction tone bursts with stimulus frequencies of $500 \mathrm{~Hz}$. In our 2 patients VEMPs were absent before surgery and, 1 month after surgery, electrical cVEMPs in the implantation side were obtained. P1 and N1 latencies were in 8-15 and 15-24 s, respectively. These results were present 6 months after that, being a testing of the activation of the vestibulo-collic reflex and, consequently, of the activation of the saccular part of the otolith organ (Fig. 5).

\section{Subjective Visual Vertical}

Subjective visual horizontal (SVH) or subjective visual vertical (SVV): In human subjects with the eyeball in a rolled position, visual perception changes in accordance with this new torsional. Systematic deviations in SVV or SVH greater than about 2 degrees, in patients with peripheral vestibular dysfunction, are taken to indicate static otolith dysfunction. Standardized measurement of the $\mathrm{SVH}$, with a dim light-bar in an otherwise totally darkened room, can give valuable diagnostic information. SVV: The visual vertical application offers the quickest and easiest to measure SVV in patients. Static SVV will be measured using the visual vertical TM (Clear Health Media, Wonga Park, VIC, Australia) application on an iPhone (Apple, Cupertino, CA, USA). The normal range of deviation is set to $2.3^{\circ}$ in either direction of the true vertical.

In this study, Static SVV was measured by using the visual vertical TM (Clear Health Media, Wonga Park, VIC, Australia) application on an iPhone (Apple, Cupertino, CA, 
USA). The normal range of deviation is set to $2.3^{\circ}$ in either direction of the true vertical. In Subject 1, a considerable improvement, (pre-surgery: $-9.6^{\circ}$ and post-surgery: $-1.3^{\circ}$ ) was obtained, with a normal value. In subject 2 , although he showed a better pre-surgery score, he also reached better results 1 month after the surgery $\left(1.1\right.$ and $\left.0.6^{\circ}\right)$

\section{Computerized Dynamic Posturography}

Computerized dynamic posturography (CDP) is an objective method of differentiating sensory, motor, and central adaptive functional impairments of balance. CDP includes the sensory organization test (SOT), the adaptation test and the limits of stability (LOS).

CDP was performed with the SMART EquiTest ${ }^{\circledR}$ CDP 8.1 version (NeuroCom ${ }^{\circledR}$, USA). It allows differentiating sensorial, motor, and central adaptive functional impairments of balance including the SOT, the adaptation test and the LOS. These tests were probably the most accurate tools to evaluate functional results of VI in real life. In both patients, immediately after activate VI, they perform better in all condition obtaining, observing a progression to normal ranges or near normal level in both cases. We can check better directional control and an increase in maximum distance excursion end point, center of gravity more centered, and evident improvement of the reaction control in both patients (Fig. 6).

\section{Dynamic Gait Index}

Dynamic gait index was developed as a clinical tool to assess gait, balance, and fall risk. It evaluates steady-state walking and also walking during more challenging tasks. In both patients, they were reaching maximum values, especially subject 1 experienced a great evolution from the beginning, going from a figure of $8 / 24$ before intervention to obtain the maximum value in the test after it $(24 / 24)$. The second patient reached initial values of $16 / 24$, and he also obtained the maximum value after the intervention $(24 / 24)$.

\section{The Time UP and Go test}

Time UP and Go test measures the time that take to stand up from a standard armchair, walk 3 meters, turn around, walk back to the chair, and sit down again. If we consider a cutoff score of $11 \mathrm{~s}$, the duration improved after surgery with normal ranges reaching a maximum of 7 $s$ in both patients.

\section{Dizziness Handicap Index}

Dizziness handicap index measures the self-perceived level of handicap associated with the symptom of dizzi- ness. In Subject 1, a clear improvement in the perception of his disability was observed, going from a severe to a mild grade. Subject 2 started from a better situation, but and also noticed a progression to better results.

With the results described above, an evident improvement in patients' quality of life was observed after a 1-year follow-up in the first patient and 7 months in the second patient. They had improved their values in the dizziness handicap index, gait in dim light and difficult spaces, and spatial orientation reaching normal ranges in tests like the dynamic gait index and Time UP and Go test. Other improvements were also observed, such as in SOT, and its static position, obtaining an adequate center of gravity in the posturography, as well as, extending the LOS.

It is also important to consider interesting details of their daily life as relevant: Both patients restored their normal activities such as walking on unlevelled terrain (beach sand), abandoning the use of walking sticks or help from other people, being able to walk on narrow sidewalks, walking in the dark, being able to carry weight, tight shoes and complete recover the daily activities without any support. Both patients used their system $24 \mathrm{~h} /$ day.

However, there are still aspects to be studied, such as the role played by the otolithic stimulus in the restoration of VOR, since a certain modulation in the results of the video head impulse test, as well as, in the spontaneous nystagmus in one of our patients was observed.

\section{Audiological Results}

Although this is not a matter of this study, both patients obtained good responses with the CI532 ${ }^{\circledR}$ CI. The subjects used alternatively both systems due to interference in the processors, and they used every day the VI system and the contralateral CI, and only used both CI when stay seated (e.g., watching TV, etc.).

\section{Discussion}

Our balance system may be activated when there is a concern for movement, but it is not active only when someone is moving, but also while maintaining a balanced posture or gait. The vestibular organ senses linear and rotational acceleration of the head during active and passive motion. These signals are necessary for bipedal locomotion, navigation, and for the coordination of eye and head movements in 3-D space. The temporal dynamics of vestibular processing in cortical structures has been poorly studied in humans. Natural otolith stimulation in subjects accelerated along the main axis shows that the 
source localization gave the cingulate sulcus visual area and the opercular-insular region as the main origin of the evoked potentials, and it is essential in the processing of acceleration intensities as sensed by the otolith organs [Ertl et al., 2017].

VI are research devices designed to rehabilitate patients with a dysfunction that impairs gaze and balance mainly in dynamic situations. Patients with BVD have a quality of life that is significantly impaired, and they can benefit from the stimulation of the otolith organ because it might have potential effects on more complex integrative behaviors, such as posture. In this research, it has been shown that a multichannel vestibular (otoliths stimulator) prosthesis can restore the vestibular sensation, as evidenced by improvements in the spatial orientation, gaze stabilization, posture, and gait. However, a key challenge is to optimize the propagation of the current beyond the otolith organ itself to the nerve branch. The otolith stimulation was performed by placing 3 electrodes in proximity of the vestibular nerve in addition to a CI.

Previous studies suggest that the implementation of a vestibular prosthesis provides partial restitution of the VOR and might also improve perception and posture in the presence of bilateral vestibular hypofunction. Good results have been reported in patients implanted with modified CI with a trifurcating array incorporating different number of electrodes per array inserted into the ampullae. Some devices were purely vestibular and others included a cochlear electrode array.

Some of these devices were conceived to act as a pacemaker that could be activated during a Meniere's crisis to regulate vestibular function in patients suffering from intractable diseases. In addition, there are new techniques that try to preserve residual hearing and, although we cannot rule out of that principle, more technical innovations must be done [Fridman et al., 2010; Golub et al., 2014; Phillips et al., 2015; Perez Fornos, 2017].

In this study, vestibular ECAP responses were obtained after electrical stimulation of the otolith organ by using a similar stimulation paradigm as in CI. The vestibular ECAP response, which has been recorded in the human vestibular end organ, displays many of the characteristics of the compound action potential recorded in the cochlea.

It was also observed that it is possible to correlate the vestibular ECAP response with the electrically oVEMPs responses through an electrode placed in the otolith organs. The ideal location of the electrode was determined by monitoring the vestibular ECAP at slightly different places during the insertion. We observed that a minimal displacement of the electrode resulted in drastic changes in the amplitude of the responses, thus stabilization of the electrode array is necessary to obtain good responses. This also indicates that the presence of vestibular ECAP correlates with electrically eoVEMPs responses, and could be used as a sign of vestibular stimulation [Ramos de Miguel et al., 2017].

Regarding the stimulation, although it is not well known how the stimulus in the vestibular organ can provide an effective response in the afferent vestibular nerve, many studies have been presented with different researches on this topic. A model of the primate labyrinth has been recently made from 3 -D reconstruction images to study the spread of prosthetic current in the inner ear and to facilitate design of electrode arrays and stimulation protocols for a VI system, which offers important data of the spread and the effect of the stimulation [Hedjoudje et al., 2019].

The role of the otoliths in human (utricle and saccule) in the global function of the vestibular function has not been widely studied and it is difficult to determine [Curthoys, 2010; Curthoys et al. 2018].

Nystagmus can be affected by gaze positions. However, there are still doubts to be solved, such as the role played by the otoliths stimulus in the restoration of VOR, since a certain modulation in the results of the video head impulse test and in the spontaneous nystagmus in one of our patients was observed. With respect to the relation with the VOR, a recent study examining the role of the otoliths in adaptation of the VOR in an animal model shows that otoliths do not contribute to the adaptation of the normal angular VOR, as it has been shown during the activation in our patients. However, the otoliths provide the main cue for gravity context-specific VOR adaptation, and also may have some effect in the spontaneous nystagmus, that also has been observed in our study [Khan et al., 2019].

In addition, it is important to consider that many authors confirmed that imbalance is a common cause of morbidity following CI. In most cases, it is not correlated with reductions in VOR gain and suggests that the underlying lesion may be isolated to other vestibular end organs as otolith organs [Rubinstein et al., 2012; Shute et al., 2018; Imai et al., 2019].

\section{Future Work}

These results indicate that electrical stimulation of the vestibular nerve, by using an otolith stimulation approach, has a significant functional impact on balance and everyday tasks such as walking, and fall prevention. The concept presented in this research has the potential to restore 
the vestibular function and could have a central role in improving the quality of life of BVL patients, but future research is needed in order to improve our knowledge:

- A new multicentre study using a new device in order to stimulate simultaneously cochlear and otolith organ will be developed in the next months (Horizon2020 Fet Open. Grant Agreement No. 801112 7. Project Acronym: BionicVEST).

- Preservation of the vestibular and hearing function will be a crucial issue and substantial efforts must be put into the design of new electrodes and surgical approaches

- Analyze the specificity of stimulation targeting the different branches of the vestibular nerve, including the saccular, utricular and semicircular canals

- Improve the specificity of the stimulus and the sensors to provide a better result.

\section{Weakness}

This study has been conducted on a much reduced number of patients, as the authorization of new procedure had to be obtained, and it was limited to 2 patients. Now, our group is authorized to include a third patient in this preliminary study and also, with a new research prototype device, a multicenter study will include 12 more patients.

\section{Conclusion}

Prosthetic implantation of the otolith organ in humans is technically feasible. Electrical stimulation might have potential effects on balance and this is stable after 1 year follow-up. This research provides new possibilities for the development of VI to improve the gravito-inertial acceleration sensation, in this case, by the otoliths stimulation.

\section{Statement of Ethics}

The current study was conducted with the approval of the Ethics Committee of our hospital (CEIm-CHUIMI 2017/956) and performed in accordance with the 1964 Helsinki Declaration and its later amendments or comparable ethical standards. All patients provided written informed consent before participating. All the procedures involving human participants were in accordance with the ethical standards of our institutional research committee.

\section{Disclosure Statement}

All authors declare that they have no conflicts of interest.

\section{Funding Sources}

No institutional or private funding was obtained for the present research.

\section{Author Contributions}

A.R.M.: design of the work; final approval of the manuscript; surgeries. A.R.dM. and J.C.F.G.: intraoperative and postoperative neurophysiological testing; analysis of data; drafting the manuscript. I.R.M. and S.B.B.: clinical tests; analysis of data; drafting the manuscript.

\section{References}

Brodal A. "Anatomy of the vestibular nuclei and their connections." In: Brodal A, Cohen Bl. editors. Vestibular System Part 1: Basic Mechanisms, D. Bagger-Sjoback. Springer Berlin Heidelberg; 1974. pp. 239-352.

Curthoys IS, Dai MJ, Halmagyi GM. Human ocular torsional position before and after unilateral vestibular neurectomy. Exp Brain Res. 1991;85(1):218-25.

Curthoys IS. A critical review of the neurophysiological evidence underlying clinical vestibular testing using sound, vibration and galvanic stimuli. Clin Neurophysiol. 2010 Feb; 121(2):132-44.

Curthoys IS, Grant JW, Burgess AM, Pastras CJ, Brown DJ, Manzari L. Otolithic Receptor Mechanisms for Vestibular-Evoked Myogenic Potentials: A Review. Front Neurol. 2018 May;9:366.
Della Santina CC, Migliaccio AA, Patel AH. A multichannel semicircular canal neural prosthesis using electrical stimulation to restore 3-d vestibular sensation. IEEE Trans Biomed Eng. 2007 Jun;54(6 Pt 1):1016-30.

Ertl M, Moser M, Boegle R, Conrad J, Zu Eulenburg P, Dieterich M. The cortical spatiotemporal correlate of otolith stimulation: vestibular evoked potentials by body translations. Neuroimage. 2017 Jul;155:50-9.

Fridman GY, Davidovics NS, Dai C, Migliaccio AA, Della Santina CC. Vestibulo-ocular reflex responses to a multichannel vestibular prosthesis incorporating a 3D coordinate transformation for correction of misalignment. J Assoc Res Otolaryngol. 2010 Sep;11(3):367-81.

Gillespie MB, Minor LB. Prognosis in bilateral vestibular hypofunction. Laryngoscope. 1999 Jan;109(1):35-41.
Goldberg JM, Fernandez C. The vestibular system. In: I. Darian-Smith, editor. Handbook of Physiology, Section 1: The Nervous System, Vol. 3, Parts 1 and 2: Sensory processes, new ed. Bethesda: American Physiological Society; 1984. pp. 977-1022.

Golub JS, Ling L, Nie K, Nowack A, Shepherd SJ, Bierer SM, et al. Prosthetic implantation of the human vestibular system. Otol Neurotol. 2014 Jan;35(1):136-47.

Halmagyi GM, Weber KP, Curthoys IS. Vestibular function after acute vestibular neuritis. Restor Neurol Neurosci. 2010;28(1):37-46.

Hedjoudje A, Hayden R, Dai C, Ahn J, Rahman M, Risi F, et al. Virtual Rhesus Labyrinth Model Predicts Responses to Electrical Stimulation Delivered by a Vestibular Prosthesis. J Assoc Res Otolaryngol. 2019 Aug;20(4): 313-39. 
Imai $\mathrm{T}$, Okumura $\mathrm{T}$, Ohta $\mathrm{Y}$, Oshima $\mathrm{K}$, Sato $\mathrm{T}$, Kamakura T, et al. Effects of cochlear implants on otolith function as evaluated by vestibulo-ocular reflex and vestibular evoked myogenic potentials. Auris Nasus Larynx. 2019 Dec;46(6):836-43.

Khan SI, Della Santina CC, Migliaccio AA. Angular vestibuloocular reflex responses in Otop1 mice. I. Otolith sensor input is essential for gravity context-specific adaptation. J Neurophysiol. 2019 Jun;121(6):2291-9.

MeshLab: An Open-Source Mesh Processing Tool. Sixth Eurographics Italian Chapter Conference; 2008. pp. 129-36.

Pauw BK, Pollak AM, Fisch U. Utricle, saccule, and cochlear duct in relation to stapedot omy. A histologic human temporal bone study. Ann Otol Rhinol Laryngol. 1991 Dec;100(12):966-70.

Perez Fornos A, Cavuscens S, Ranieri M, van de Berg R, Stokroos R, Kingma H, et al. The vestibular implant: A probe in orbit around the human balance system. J Vestib Res. 2017; 27(1):51-61.
Phillips JO, Ling L, Nie K, Jameyson E, Phillips $\mathrm{CM}$, Nowack AL, et al. Vestibular implantation and longitudinal electrical stimulation of the semicircular canal afferents in human subjects. J Neurophysiol. 2015 Jun;113(10): 3866-92.

Ramos de Miguel A, Falcon Gonzalez JC, Ramos Macias A. Vestibular Response to Electrical Stimulation of the Otolith Organs. Implications in the Development of a Vestibular Implant for the Improvement of the Sensation of Gravitoinertial Accelerations. J Int Adv Otol. 2017 Aug;13(2):154-61.

Rosset A, Spadola L, Ratib O. OsiriX: An OpenSource Software for Navigating in Multidimensional DICOM Images. J Digit Imaging. 2004 Sep;17(3):205-16.

Rubinstein JT, Bierer S, Kaneko C, Ling L, Nie K, Oxford $\mathrm{T}$, et al. Implantation of the semicircular canals with preservation of hearing and rotational sensitivity: a vestibular neurostimulator suitable for clinical research. Otol Neurotol. 2012 Jul;33(5):789-96.
Shute WG, McOwan B, O'Leary SJ, Szmulewicz D. The Early Postoperative Effects of Cochlear Implantation on Horizontal Semicircular Canal Function. Otol Neurotol. 2018 Aug; 39(7):e524-31.

Sprinzl GM, Riechelmann H. Current trends in treating hearing loss in elderly people: a review of the technology and treatment options - a mini-review. Gerontology. 2010; 56(3):351-8.

Strupp M, Kim JS, Murofushi T, Straumann D, Jen JC, Rosengren SM, et al. Bilateral vestibulopathy: Diagnostic criteria Consensus document of the Classification Committee of the Bárány Society. J Vestib Res. 2017;27(4):17789.

Ward BK, Agrawal Y, Hoffman HJ, Carey JP, Della Santina CC. Prevalence and impact of bilateral vestibular hypofunction: results from the 2008 US National Health Interview Survey. JAMA Otolaryngol Head Neck Surg. 2013 Aug;139(8):803-10. 